\title{
COnditions FOR Sylow 2-SUBGROUPS OF THE FIXED POINT SUBGROUP IMPLYING SOLUBILITY
}

\author{
Antonio Beltrán \\ Departamento de Matemáticas, \\ Universidad Jaume I, 12071 Castellón, Spain \\ e-mail: abeltran@mat.uji.es \\ Changguo Shao \\ School of Mathematical Science, \\ University of Jinan, 250022, Shandong, China, \\ e-mail: shaoguozi@163.com
}

\begin{abstract}
Let $A$ and $G$ be finite groups and suppose that $A$ acts via automorphisms on $G$ with $(|A|,|G|)=1$. We study how certain conditions on the Sylow 2-subgroups of the fixed point subgroup of the action, $\mathbf{C}_{G}(A)$, may imply the non-simplicity or solubility of $G$.
\end{abstract}

Keywords. Finite groups, Sylow subgroups, Coprime action, Group action on groups.

Mathematics Subject Classification (2010): 20D20, 20 D15.

\section{Introduction}

The Sylow 2-subgroups play a crucial role in the internal structure of finite groups, and especially, of non-abelian simple groups. For instance, as a consequence of Burnside's normal $p$-complement theorem (see $[6,7.2 .1]$ ) it follows that when the Sylow 2-subgroups of a finite group $G$ are cyclic, then $G$ necessarily possesses normal 2-complement, and by appealing to the Feit-Thompson Theorem, it turns out that $G$ is soluble. Likewise, another celebrated non-simplicity criterium concerning Sylow 2-subgroups is the Brauer-Suzuki Theorem [2] (or more general, Glauberman $\mathbf{Z}^{*}$-theorem). It claims that if a finite group $G$ has a generalized (or ordinary) quaternion Sylow 2-subgroup and no non-trivial normal subgroups of odd order, then $G$ has a centre of order 2. In particular, $G$ cannot be simple. We shall make use of these facts without further reference. 
Another important and necessary result is the classification of the finite simple groups having elementary abelian or dihedral Sylow 2-subgroups, which were completely determined in [8] and [5], respectively.

Now, let $G$ and $A$ be finite groups of relatively prime orders such that the group $A$ acts on $G$. Under this coprime action hypothesis, we investigate what information on the Sylow 2-subgroups of the fixed point subgroup, $\mathbf{C}_{G}(A)$, may provide solubility properties in the whole group $G$. For example, it is known that if these 2-subgroups are trivial, that is, when $\mathbf{C}_{G}(A)$ has odd order, then $G$ is soluble ([7]). The same happens when the Sylow 2-subgroup of $\mathbf{C}_{G}(A)$ is a direct factor of $\mathbf{C}_{G}(A)$, and this is also a consequence of the main theorem of [7]. On the other hand, several solubility conditions of $G$ have been given under the assumption that $G$ has exactly one $A$-invariant Sylow $p$-subgroup for certain prime $p([1])$. Going one step further, one may wonder whether we can get the solubility of $G$ from the fact that the Sylow 2-subgroups of $\mathbf{C}_{G}(A)$ have a specific structure, that is, they are either cyclic, elementary abelian, dihedral or generalized quaternion groups. The answer is negative and it is not hard to find examples of simple groups acted on by groups of coprime order satisfying such conditions. However, we shall show how these conditions strongly limit both the simple cases and the non-soluble structure of the group. Our results are based on the Classification Theorem of Finite Simple Groups (CFSG) and we will use the standard notation appearing in [3]. All groups are supposed to be finite.

Theorem 1.1. Assume that a group $A$ acts coprimely on a group $G$ and that $\mathbf{C}_{G}(A)$ has a cyclic or elementary abelian Sylow 2-subgroup. Then $G$ is soluble if and only if $G$ has no composition factor isomorphic to one of the following groups:

1) $\operatorname{PSL}\left(2,2^{n}\right)$, with $n \geq 2 ; \operatorname{PSL}\left(2, q^{n}\right)$, where $q$ is a prime power such that $q \equiv 3,5(\bmod 8)$ and $n \geq 1$, with $q^{n} \neq 3$,

2) $\mathrm{Sz}\left(2^{n}\right)$ with $n \geq 2$,

3) ${ }^{2} \mathrm{G}_{2}\left(3^{n}\right)$ with $n \geq 2$,

4) the sporadic simple group $\mathrm{J}_{1}$

Theorem 1.2. Assume that a group $A$ acts coprimely on a group $G$ and that the Sylow 2-subgroups of $\mathbf{C}_{G}(A)$ are generalized quaternion. Then $G$ is soluble if and only if $G$ has no composition factor isomorphic to one of the following groups:

1) $\operatorname{PSL}\left(2,2^{n}\right)$, with $n \geq 2 ; \operatorname{PSL}\left(2, q^{n}\right)$, where $q$ is a prime power such that $q \equiv 3,5(\bmod 8)$ and $n \geq 1$, with $q^{n} \neq 3$,

2) $\mathrm{Sz}\left(2^{n}\right)$ with $n \geq 2$,

3) the alternating group $\mathrm{A}_{7}$, 
5) $\operatorname{PSU}\left(3,2^{n}\right)$ with $n \geq 2$.

Theorem 1.3. Assume that a group $A$ acts coprimely on a group $G$ and that the Sylow 2-subgroups of $\mathbf{C}_{G}(A)$ are dihedral groups. Then $G$ is soluble if and only if $G$ has no composition factor isomorphic to one of the following groups:

1) $\operatorname{PSL}(2, q)$, with $q$ a prime power, $q>3$,

2) $\mathrm{Sz}\left(2^{n}\right)$ with $n \geq 2$,

3) the alternating group $\mathrm{A}_{7}$.

\section{Preliminaries}

In this section, we present some results that we need for our purposes. Suppose that a finite group $G$ is acted on via automorphims by another finite group $A$ satisfying $(|A|,|G|)=1$. Under this coprime action hypothesis and for every prime $p$, the group $A$ naturally acts on the set of Sylow $p$-subgroups of $G$, there always exist $A$-invariant Sylow $p$-subgroups in $G$, and any two of them are conjugate by some element in the fixed point subgroup $C=\mathbf{C}_{G}(A)$. Furthermore, for any $A$-invariant Sylow subgroup $P$ of $G$, we have that $P \cap C$ is a Sylow $p$-subgroup of $C$. We refer the non-familiarized reader to [6, Chapter 8] for a detailed presentation of the basic properties of coprime action. We only state here some lemmas, which will be frequently used.

Lemma 2.1. $[6,8.2 .2]$ Let $A$ acts coprimely on $G$. Let $N$ be an A-invariant normal subgroup of $G$. Suppose that the action of $A$ on $N$ is coprime, then $\mathbf{C}_{G / N}(A)=\mathbf{C}_{G}(A) N / N$.

Lemma 2.2. [1, Lemma 2.3] Suppose that a finite group $A$ acts on a finite group $G=H_{1} \times \cdots \times H_{n}$, such that $H_{i}^{a} \in\left\{H_{1}, \ldots, H_{n}\right\}$ for all $a \in A$ and $i \in\{1, \ldots, n\}$. Assume further that $A$ acts transitively on $\left\{H_{1}, \ldots, H_{n}\right\}$. Let $H=H_{1}$ and $B=\mathbf{N}_{A}(H)$. Then $\mathbf{C}_{G}(A) \cong \mathbf{C}_{H}(B)$.

In the above lemma, the orders of $A$ and $G$ need not have relatively prime orders. However, in our arguments this will be applied under the coprime action hypothesis and in the following situation.

Lemma 2.3. Suppose that a finite group $A$ acts coprimely on a finite group $G$ and that $G$ does not have any proper $A$-invariant normal subgroup. Then $G=$ $H_{1} \times \ldots \times H_{n}$, where $H_{i}$ are isomorphic simple groups and $\mathbf{C}_{G}(A) \cong \mathbf{C}_{H}(B)$, where $H=H_{1}$ and $B=\mathbf{N}_{A}(H)$.

Proof. Since $G$ has no $A$-invariant normal subgroups, then $G$ is a minimal normal subgroup of $G A$, the semidirect product of $G$ and $A$, and accordingly, $G$ is the direct product of isomorphic simple groups, say $G=H_{1} \times \ldots \times H_{n}$. Moreover, on can easily prove that $A$ must act transitively on the set $\left\{H_{1}, \ldots, H_{n}\right\}$, so we apply Lemma 2.2 and the result follows. 
The next result contains certain known lists of non-abelian simple groups whose Sylow 2-subgroups have a concrete structure. We remark that when we say dihedral group, we also include the case $C_{2} \times C_{2}$.

Lemma 2.4. Let $G$ be a non-abelian simple group with a Sylow 2-subgroup P.

a) If $P$ is elementary abelian, then $G$ is isomorphic to $\operatorname{PSL}(2, q)$, with $q$ a prime power, $q>3, q \equiv 3,5(\bmod 8)$ or $q=2^{n}, \mathrm{~J}_{1}$ or ${ }^{2} \mathrm{G}_{2}\left(3^{n}\right)$ with $n=2 m+1$.

b) If $P$ is a dihedral group, then $G$ is isomorphic to $\operatorname{PSL}(2, q)$, with $q$ a prime power, $q>3$ odd, or the alternating group $\mathrm{A}_{7}$.

In particular, when $P$ is an elementary abelian group of order 4 , then $G$ is isomorphic to $\operatorname{PSL}(2, q)$, with $q$ a prime power $q>3, q \equiv 3,5(\bmod 8)$ or $q=4$.

Proof. Part (a) is [4, Theorem, p. 485] and part (b) is [4, Theorem, p. 462]. Assume now that $P$ is elementary abelian of order 4 . By (a), $G$ is isomorphic to $\operatorname{PSL}(2, q), q>3, q \equiv 3,5(\bmod 8)$ or $q=2^{n}$, $\mathrm{J}_{1}$ or ${ }^{2} \mathrm{G}_{2}\left(3^{n}\right)$, with $n$ odd. However, any Sylow 2-subgroup of $\mathrm{J}_{1}$ has order 8 (see [3]). The group ${ }^{2} \mathrm{G}_{2}\left(3^{n}\right)$ satisfies $\left|{ }^{2} \mathrm{G}_{2}\left(3^{n}\right)\right|=3^{3 n}\left(3^{3 n}+1\right)\left(3^{n}-1\right)$ with $n$ odd, and notice that $4 \mid 3^{3 n}+1$ and $2 \mid 3^{n}-1$, so $|P| \geq 8$. We also know that the Sylow 2-subgroups of $\operatorname{PSL}\left(2,2^{n}\right)$ have order $2^{n}$, so we get $n=2$. On the other hand, by order calculations, it is easily seen that every Sylow 2-subgroup of $\operatorname{PSL}(2, q)$ with $q>3, q \equiv 3,5(\bmod 8)$ is elementary abelian of order 4 . We conclude that $G$ is isomorphic to $\operatorname{PSL}(2, q), q>3, q \equiv 3,5(\bmod 8)$ or $q=4$, and the last part of the lemma is proved.

We need a description of the normal structure and the quotient structure of the generalized quaternion groups and of the dihedral groups of order $2^{n}$, with $n \geq 3$. We compile all the specific information that we shall use later and provide a proof of it because, in spite of the fact that the properties are known, we have not been able to find in the literature a result which collects all those that we exactly need.

Lemma 2.5. Let $G=Q_{2^{n}}=\left\langle a, b \mid a^{2^{n-1}}=1, b^{4}=1, a^{b}=a^{-1}\right\rangle$ be the generalized quaternion group of order $2^{n}$ with $n \geq 3$ and let $N$ be a non-trivial proper normal subgroup of $G$. Then

a) If $N \leq\langle a\rangle$, then either

a.1) $N=\left\langle a^{2^{n-2}}\right\rangle=\mathbf{Z}(G)$ and $G / N \cong D_{2^{n-1}}$, the dihedral group of order $2^{n-1} ;$ or

a.2) $\mathbf{Z}(G)<N<\left\langle a^{2}\right\rangle$ and $G / N \cong Q_{2^{i}}$ with $3 \leq i \leq n-2$, or $N=\left\langle a^{2}\right\rangle$ and $G / N \cong C_{2} \times C_{2}$;

a.3) $N=\langle a\rangle$ and $G / N \cong C_{2}$.

b) If $N \not\langle a\rangle$, then $N$ is a generalized quaternion group and $G / N \cong C_{2}$. 
Proof. Let $H=\langle a\rangle$. We divide the proof into two cases depending on whether $N \leq H$ or not. Suppose first that $N \not H$. Then $G=H N$ and $G / H \cong$ $N /(H \cap N) \cong C_{2}$. This shows that $a^{2} \in N$. Since $N$ must contain some $a^{i} b$ for some integer $1 \leq i \leq 2^{n-1}$, we obtain $N=\left\langle a^{2}, a^{i} b\right\rangle$. If $2 \nmid i$, then $a b \in N$ and thus $N=\left\langle a^{2}, a b\right\rangle$. If $2 \mid i$, then $b \in N$ and $N=\left\langle a^{2}, b\right\rangle$. In both cases $N$ is a generalized quaternion group and $|G / N|=2$.

Suppose now that $N \leq H$. We trivially have one of the three possibilities: $N=\mathbf{Z}(G), \mathbf{Z}(G)<N<H$, or $N=H$. In each case, we have $G / N \cong D_{2^{n-1}}$, $G / N \cong Q_{2^{i}}$ with $3 \leq i \leq n-2$, or $C_{2} \times C_{2}$, respectively.

The next lemma is elementary and we omit its proof.

Lemma 2.6. Let $G=D_{2^{n}}$ be the dihedral group of order $2^{n}$. Then every normal subgroup $N$ of $G$ is cyclic or dihedral. Furthermore, for every $n \geq 3$, if $|N|=2^{n-1}$ then $G / N \cong C_{2}$ and if $|N|=2^{i}$ with $i \leq n-2$, then $G / N \cong D_{2^{n-i}}$.

Remark 2.7. The following fact is well known (see [3]). The list of groups of Lie type which are non-simple is the following: $\mathrm{A}_{1}(2) \cong \mathrm{PSL}(2,2) \cong \mathrm{S}_{3} ; \mathrm{A}_{1}(3) \cong$ $\operatorname{PSL}(2,3) \cong \mathrm{A}_{4} ;{ }^{2} \mathrm{~A}_{2}(2) \cong \operatorname{PSU}(3,2)$, which is a Frobenius group with complement $Q_{8} ;{ }^{2} \mathrm{~B}_{2}(2) \cong \mathrm{Sz}(2)$, the Frobenius group of order $20 ; \mathrm{B}_{2}(2) \cong \mathrm{S}_{6} ; \mathrm{G}_{2}(2)$ which has order 12096 and the derived subgroup is isomorphic to $\operatorname{PSU}(3,3)$; ${ }^{2} \mathrm{G}_{2}(3)$ whose derived subgroup is isomorphic to $\operatorname{PSL}\left(2,2^{3}\right) ;{ }^{2} \mathrm{~F}_{4}(2)$ whose derived subgroup is the Tits (simple) group. In particular, we observe that in this list only the groups $\operatorname{PSL}(2,2)$ and $\mathrm{Sz}(2)$ have cyclic Sylow 2-subgroups (of orders 2 and 4 , respectively), only the groups $\operatorname{PSL}(2,2), \operatorname{PSL}(2,3)$ and ${ }^{2} \mathrm{G}_{2}(3)$ have elementary abelian Sylow 2-subgroups (of orders 2, 4 and 8, respectively), and $\operatorname{PSU}(3,2)$ is the only group having (generalized) quaternion Sylow 2-subgroups. Moreover, none of the groups of the list has a dihedral Sylow 2-subgroup of order greater than 4 .

\section{Proofs}

Before proving our main results we need to determine the non-abelian simple groups satisfying certain coprime action conditions. We prove first a property about coprime action on simple groups appealing to the CFSG.

Lemma 3.1. Assume that a group $A$ acts non-trivially and coprimely on a non-abelian simple group $G$. Then $G$ is a simple group of Lie type, say $G=$ $G\left(q^{r}\right)$, defined over the field with $q^{r}$ elements, where $q$ is a prime power and $r=\left|A / \mathbf{C}_{A}(G)\right|$. Moreover, $\mathbf{C}_{G}(A) \cong G(q)$, the simple group of Lie type of the same type as $G$, but defined on the field of $q$ elements.

Proof. It is known that the alternating groups and the 26 sporadic simple groups do not admit a non-trivial coprime automorphism. In fact, this can be easily checked, for instance in [3], that if $G$ is any of such groups, then every prime divisor of the order of its outer automorphism group, $\operatorname{Out}(G)$, also divides $|G|$. Hence, by using the CFSG, it follows that $G$ is a simple group of Lie type defined 
over some finite field $F$. Now, we consider the action of $\bar{A}:=A / \mathbf{C}_{A}(G)$ on $G$, where $\mathbf{C}_{A}(G)$ denotes the kernel of the action of $A$ on $G$. It is clear that $\bar{A}$ also acts non-trivially (and coprimely) on $G$. Furthermore, since this action is faithful, by replacing $\bar{A}$ by some conjugate in $\operatorname{Aut}(G)$, we may assume that $\bar{A}$ is an automorphism group of $G$ induced by some automorphism group of $F$. Now, if $|\bar{A}|=r$, it follows that $|F|=q^{r}$ for some prime power $q$. If we write $G=G\left(q^{r}\right)$, the simple group of Lie type, then $\mathbf{C}_{G}(A)=\mathbf{C}_{G}(\bar{A}) \cong G(q)$, the (non necessarily simple) Lie group of the same type as $G$, but defined over the field of $q$ elements.

Theorem 3.2. Assume that a group $A$ acts non-trivially and coprimely on a non-abelian simple group $G$ and let $P$ be a Sylow 2-subgroup of $\mathbf{C}_{G}(A)$.

a) If $P$ is cyclic, then $G \cong \operatorname{PSL}\left(2,2^{n}\right)$, with $n \geq 2$ or $G \cong \operatorname{Sz}\left(2^{n}\right)$ with $n \geq 2$.

b) If $P$ is elementary abelian, then $G$ is isomorphic to one of these groups: $\operatorname{PSL}\left(2,2^{n}\right)$, with $n \geq 2 ; \operatorname{PSL}\left(2, q^{n}\right)$, where $q$ is a prime power such that $q \equiv 3,5(\bmod 8)$ and $n \geq 1$, with $q^{n} \neq 3$; or ${ }^{2} \mathrm{G}_{2}\left(3^{n}\right)$ with $n \geq 2$.

Proof. We prove a) and b) at the same time. We reason by induction on $|G A|$, where $G A$ denotes the semidirect product of $G$ by $A$. Let $C:=\mathbf{C}_{G}(A)$. By applying Lemma 3.1, we can assume that $G$ is a simple group of Lie type, say $G=G\left(q^{r}\right)$, where $q$ is a prime power and $r=\left|A / \mathbf{C}_{A}(G)\right|$. Also, $C \cong G(q)$, the simple group of the same Lie type, defined on the field of $q$ elements. We distinguish whether $C$ is simple or not. If $C$ is non-abelian simple group, then we know that $C$ cannot have cyclic Sylow 2-subgroups, and if $C$ has an elementary abelian Sylow 2-subgroup, by Lemma 2.4(a) we have $C \cong \operatorname{PSL}(2, q)$, where $q \equiv 3,5(\bmod 8)$ or $q=2^{m}$ or ${ }^{2} \mathrm{G}_{2}\left(3^{m}\right), m=2 k+1$. Hence $G \cong \operatorname{PSL}\left(2, q^{r}\right)$, where $q \equiv 3,5(\bmod 8)$ or $q=2^{m}$, or ${ }^{2} \mathrm{G}_{2}\left(3^{m r}\right)$. Furthermore, since $2 \nmid r$, notice that $q^{r} \equiv 3,5(\bmod 8)$.

Assume now that $C$ is non-simple and let $P$ be a Sylow 2-subgroup of $C$. According to Remark 2.7, when $P$ is cyclic then $C \cong \operatorname{PSL}(2,2)$ or $\mathrm{Sz}(2)$, and when $P$ is elementary abelian then $C$ is isomorphic to $\operatorname{PSL}(2,2), \operatorname{PSL}(2,3)$ or ${ }^{2} \mathrm{G}_{2}(3)$. In the first case, we have $G \cong \operatorname{PSL}\left(2,2^{r}\right)$ or $\operatorname{Sz}\left(2^{r}\right)$ with $r \geq 2$, so (a) is proved. In the second case, $G \cong \operatorname{PSL}\left(2,2^{r}\right), \operatorname{PSL}\left(2,3^{r}\right)$, or ${ }^{2} \mathrm{G}_{2}\left(3^{r}\right)$. Moreover, in the case $G \cong \operatorname{PSL}\left(2,3^{r}\right)$, as $r$ is odd, then $3^{r} \equiv 3(\bmod 8)$. By taking into account the above paragraph, we conclude that $G$ is isomorphic to one of the groups listed in (b).

We are ready to prove the main results.

Proof of Theorem 1.1. We only need to prove the "only if" part of the theorem. Let $C:=\mathbf{C}_{G}(A)$ and let $\Omega=\{\operatorname{PSL}(2, q)$, where $q \equiv 3,5(\bmod 8)$ or $q=$ $2^{n}, \mathrm{Sz}\left(2^{n}\right), \mathrm{J}_{1},{ }^{2} \mathrm{G}_{2}\left(3^{n}\right)$, with $\left.n \geq 2\right\}$. Assume that $G$ has no composition factor isomorphic to one of the groups in $\Omega$. We argue by minimal counterexample, so let us take $G$ and $A$ satisfying the hypotheses with $G$ non-soluble and $|G A|$ as small as possible, where as usual $G A$ denotes the semidirect product. 
Let $N$ be a proper $A$-invariant normal subgroup of $G$. Then $\mathbf{C}_{N}(A)=$ $C \cap N \unlhd C$ and it is clear then that every Sylow 2-subgroup of $\mathbf{C}_{N}(A)$ is normal in some Sylow 2-subgroup of $C$, so in particular, it is cyclic or elementary abelian too. As $N$ also has no composition factor which belongs to $\Omega$, the minimality implies that $N$ is soluble. Thus every proper $A$-invariant normal subgroup of $G$ is soluble.

Let $N$ be a maximal $A$-invariant normal subgroup of $G$ and suppose that $N \neq 1$. By Lemma $2.1, \mathbf{C}_{G / N}(A)=C N / N \cong C /(C \cap N)$, so $\mathbf{C}_{G / N}(A)$ has cyclic or elementary abelian Sylow 2-subgroups. Since $G / N$ cannot have any composition factor isomorphic to any group in $\Omega$, the minimality guarantees that $G / N$ is soluble, and then the solubility of $N$ forces $G$ to be soluble, a contradiction. As a result, $N=1$, that is, $G$ can be assumed to have no proper $A$-invariant normal subgroup. We shall prove that $G$ is non-abelian simple.

According to Lemma 2.3, we write $G=H_{1} \times \ldots \times H_{n}$ with $C \cong \mathbf{C}_{H}(B)$, where $H=H_{1}$ and $B=\mathbf{N}_{A}(H)$. Also, we claim that the action of $B$ on $H$ cannot be trivial. Otherwise, $C \cong H$ and thus the Sylow 2-subgroups of $H$ are cyclic or elementary abelian. Then Lemma 2.4(a) would imply that $H$ is isomorphic to $\operatorname{PSL}(2, q), q>3, q \equiv 3,5(\bmod 8)$ or $q=2^{n}, \mathrm{~J}_{1}$ or ${ }^{2} \mathrm{G}_{2}\left(3^{n}\right)$ with $n$ odd, which contradicts the hypotheses of the theorem. Once we have proved that the action of $B$ on $H$ is non-trivial (and coprime), we can apply Lemma 3.1 to obtain $H=H\left(q^{t}\right)$ and $\mathbf{C}_{H}(B)=H(q)$, both simple groups of the same Lie type, where $q^{t}$ and $q$ are corresponding orders of the underlying fields, and $t=\left|B / \mathbf{C}_{B}(H)\right|$. Now, the fact that $C \cong \mathbf{C}_{H}(B)$ forces that $\mathbf{C}_{H}(B)$ has cyclic or elementary abelian Sylow 2-subgroups. Since $H$ has no composition factor in $\Omega$, if $|H B|<|G A|$, we get that $H$ is soluble by minimality. This contradiction shows that $G$ is non-abelian simple.

Finally, we distinguish whether the action of $A$ on $G$ is trivial or not. When it is trivial, we certainly have a contradiction by Lemma 2.2(a). On the contrary, if the action is non-trivial, we can apply Theorem 3.2 to obtain that $G$ is isomorphic to $\operatorname{PSL}\left(2,2^{r}\right), \operatorname{PSL}\left(2, q^{r}\right)$, with $q \equiv 3,5(\bmod 8), \mathrm{Sz}\left(2^{r}\right)$ or ${ }^{2} \mathrm{G}_{2}\left(3^{r}\right)$, contradicting the hypotheses too.

Remark 3.3. We show that none of the simple groups appearing in the statement of Theorem 1.1 can be eliminated, and for this reason this theorem cannot be improved. Let $H$ be any of the simple groups listed in Theorem 1.1 and choose $p$ and $q$ to be two distinct primes with $p$ odd and $(q,|H|)=1$. Let $A=C_{q}$ act trivially on $H$ and cyclically on the direct product of $q$ copies of $C_{p}$, say $K=C_{p} \times \ldots \times C_{p}$. Let us consider the corresponding coprime action of $A$ on $G=H \times K$. We clearly have $\mathbf{C}_{G}(A) \cong H \times C_{p}$ and thus the Sylow 2-subgroups of $\mathbf{C}_{G}(A)$ are the same as those of $H$. Therefore, $G$ is a non-soluble group with a composition factor isomorphic to $H$ and the Sylow 2-subgroups of $\mathbf{C}_{G}(A)$ are cyclic or elementary abelian.

Proof of Theorem 1.2. We argue by counterexample of minimal order. Let us take $G$ and $A$ satisfying the hypotheses with $G$ non-soluble and $|G A|$ as small as possible. We divide the proof into several steps. 
Step 1. Every proper $A$-invariant normal subgroup of $G$ is soluble.

Let $N$ be an $A$-invariant normal subgroup of $G$. Then $\mathbf{C}_{N}(A)=C \cap N \unlhd C$, so every Sylow 2-subgroup of $\mathbf{C}_{N}(A)$ is normal in certain Sylow 2-subgroup of $C$. By applying Lemma 2.5, we deduce that a Sylow 2-subgroup $P$ of $\mathbf{C}_{N}(A)$ is cyclic (possibly trivial) or generalized quaternion. Assume first that $P$ is cyclic. Suppose that $N$ is non-soluble and let $W$ be the soluble radical of $N$. Take $M / W>1$ a chief factor of $G A$ with $M \leq N$. Notice that $\mathbf{C}_{M / W}(A) \leq$ $\mathbf{C}_{N / W}(A)$, and then the Sylow 2-subgroups of $\mathbf{C}_{M / W}(A)$ are cyclic. By Lemma 2.3 , we can write $M / W=D_{1} \times \cdots \times D_{r}$, where $D_{i}$ are non-soluble isomorphic simple groups, and $\mathbf{C}_{M / W}(A) \cong \mathbf{C}_{D}(B)$ where $D=D_{1}$ and $B:=\mathbf{N}_{A}(D)$. All these facts imply that the Sylow 2-subgroups of $\mathbf{C}_{D}(B)$ are cyclic too. We note that the action of $B$ on $D$ cannot be trivial, otherwise the simple group $D$ would have a cyclic Sylow 2-subgroup. Thus we can apply Theorem 3.2(a) to obtain $D \cong \operatorname{PSL}\left(2,2^{n}\right)$ or $\mathrm{Sz}\left(2^{n}\right)$. As a result, $G$ has a composition factor isomorphic to one of those groups, a contradiction. Therefore, $N$ is soluble, as we wanted to prove. When $P$ is generalized quaternion, the minimality of $G A$ implies that $N$ is soluble too.

Step 2. $G$ has no proper $A$-invariant normal subgroup.

Let $N$ be a maximal $A$-invariant normal subgroup of $G$ and assume that $N \neq 1$. By step 1 , notice that $G / N$ cannot be soluble. As $G / N$ has no proper $A$-invariant normal subgroups, we may apply Lemma 2.3 to write $G / N=S_{1} \times$ $\cdots \times S_{t}$, where $S_{i}$ are isomorphic (non-abelian) simple groups, and $\mathbf{C}_{G / N}(A) \cong$ $\mathbf{C}_{S}(B)$, where $S=S_{1}$ and $B=\mathbf{N}_{A}(S)$. We claim that the action of $B$ on $S$ is non-trivial. Suppose that this action is trivial. Since $\mathbf{C}_{G / N}(A)=C N / N$ and $\mathbf{C}_{S}(B)=S$, we deduce that $C /(C \cap N) \cong S$. By applying Lemma 2.5 , it follows that any Sylow 2-subgroup $P$ of $S$ is cyclic, dihedral or generalized quaternion. However, as $S$ is a non-abelian simple group, $P$ cannot be cyclic or generalized quaternion. Then $P$ must be dihedral, so $S$ is isomorphic to $\operatorname{PSL}(2, q)$, with $q>3$ odd, or $\mathrm{A}_{7}$, by Lemma 2.4(b). This provide in $G$ a composition factor isomorphic to $\operatorname{PSL}(2, q)$, or $\mathrm{A}_{7}$, a contradiction. We conclude that $B$ acts nontrivially on $S$ as claimed.

Therefore, we can apply Lemma 3.1 and we have $S=S\left(q^{r}\right)$, the simple group of Lie type defined over a field of $q^{r}$ elements, and $\mathbf{C}_{S}(B)=S(q)$, of the same type. Since $\mathbf{C}_{G / N}(A) \cong C /(C \cap N)$, again by Lemma 2.5, the Sylow 2-subgroup of $\mathbf{C}_{G / N}(A)$ are cyclic of order 2, dihedral or generalized quaternion. Then a Sylow 2-subgroup $T$ of $\mathbf{C}_{S}(B)$ is also cyclic of order 2, dihedral group or generalized quaternion, and we distinguish each one of the possibilities.

Suppose first that $T$ is cyclic of order 2 . Of course, $\mathbf{C}_{S}(B)$ cannot be simple, in fact, Remark 2.7 yields $\mathbf{C}_{S}(B) \cong \operatorname{PSL}(2,2)$. Consequently, $S \cong \operatorname{PSL}\left(2,2^{r}\right)$, contradicting the hypotheses. Suppose now that $T \cong Q_{2^{n}}$ with $n \geq 3$. Then the minimality implies that $S$ is soluble, a contradiction too. Finally, we suppose that $T$ is a dihedral group and distinguish two cases depending on whether $\mathbf{C}_{S}(B)$ is simple or not. When $\mathbf{C}_{S}(B)$ is simple, by Lemma $2.4(\mathrm{~b})$, we have 
$\mathbf{C}_{S}(B) \cong \operatorname{PSL}(2, q)$ where $q>3$ is odd. We deduce that $S \cong \operatorname{PSL}\left(2, q^{r}\right)$ and this shows that $G$ has a composition factor isomorphic to $\operatorname{PSL}\left(2, q^{r}\right)$, a contradiction. If $\mathbf{C}_{S}(B)$ is not a simple group (of Lie type), since every group appearing in Remark 2.7 has no Sylow 2-subgroup isomorphic to a dihedral group except $\operatorname{PSL}(2,3)$ (whose Sylow 2-subgroup is $\left.C_{2} \times C_{2}\right)$, this leads to $G \cong \operatorname{PSL}\left(2,3^{r}\right)$ with $r>1$, which also provides a contradiction. Therefore, $G$ has no proper $A$-invariant normal subgroup.

Step 3. Final contradiction.

By Step 2 and Lemma 2.3, we can write $G=H_{1} \times \cdots \times H_{n}$, where the $H_{i}$ are isomorphic (non-abelian) simple groups, and $C \cong \mathbf{C}_{H}(B)$ with $H=H_{1}$ and $B=\mathbf{N}_{A}(H)$. In particular, $\mathbf{C}_{H}(B)$ possesses a generalized quaternion Sylow 2-subgroup. Now, if $H<G$, by minimality we get that $H$ is soluble, a contradiction, so $G$ is a non-abelian simple group. Furthermore, by Lemma 3.1, $G$ is a simple group of Lie type, say $G=G\left(q^{r}\right)$, defined over the finite field of $q^{r}$ elements with $r=\left|A / \mathbf{C}_{A}(G)\right|$, and $C=G(q)$. Since the Sylow 2-subgroups of $C$ are generalized quaternion, $C$ cannot be a non-abelian simple group. Hence, according to Remark 2.7, we conclude that $C$ must be isomorphic to exactly $\operatorname{PSU}(3,2)$, and so $G \cong \operatorname{PSU}\left(3,2^{r}\right)$, which is the final contradiction.

Remark 3.4. It may seem unusual that the group $\mathrm{A}_{7}$, which does not admit a non-trivial coprime action, may be a composition factor of a group $G$ satisfying the conditions of Theorem 1.2. However, it is enough to consider $H=2 . \mathrm{A}_{7}$, the central extension of $\mathrm{A}_{7}$ by a cyclic group of order 2, which has generalized quaternion Sylow 2-subgroups of order 16. Now, if we take $A \cong C_{q}$ and $K \cong$ $C_{p} \times \ldots \times C_{p}$ as in Remark 3.3, and let $A$ act (coprimely and non-trivially) on $G=H \times K$, we clearly have $\mathbf{C}_{G}(A) \cong H \times C_{p}$, whose Sylow 2-subgroups are generalized quaternion groups.

Proof of Theorem 1.3. We only need to prove the converse direction and argue by minimal counterexample. Suppose that $G$ and $A$ satisfy the hypotheses with $G$ non-soluble and $|G A|$ as small as possible.

Step 1. Every proper $A$-invariant normal subgroup of $G$ is soluble.

Let $N$ be an $A$-invariant normal subgroup of $G$. As $\mathbf{C}_{N}(A)=C \cap N \unlhd C$, then every Sylow 2-subgroup $P$ of $\mathbf{C}_{N}(A)$ is normal in certain Sylow 2-subgroup of $C$. By Lemma 2.6, we have that $P$ must be cyclic (possibly trivial) or dihedral. Assume first that $P$ is cyclic and suppose that $N$ is non-soluble. Let $W$ be the soluble radical of $N$ and let $M / W$ be a chief factor of $G A$ with $M \leq N$. As $\mathbf{C}_{M / W}(A) \leq \mathbf{C}_{N / W}(A)$, every Sylow 2-subgroup of $\mathbf{C}_{M / W}(A)$ is cyclic. We can apply Lemma 2.3 and write $M / W=D_{1} \times \cdots \times D_{r}$, where $D_{i}$ are isomorphic simple groups, and $\mathbf{C}_{M / W}(A) \cong \mathbf{C}_{D}(B)$, where $D=D_{1}$ and $B=\mathbf{N}_{A}(D)$. We deduce that a Sylow 2-subgroup of $\mathbf{C}_{D}(B)$ is cyclic too. Observe that the action of $B$ on $D$ cannot be trivial, and hence, Theorem 3.2 gives $D \cong \operatorname{PSL}\left(2,2^{n}\right)$ or $\mathrm{Sz}\left(2^{n}\right)$. This forces $G$ to have a composition factor isomorphic to one of those 
groups, a contradiction. In this case, $N$ is soluble. On the other hand, if we assume that $P$ is dihedral, then $N$ is soluble too by minimality.

Step 2. $G$ has no proper $A$-invariant normal subgroup.

Let $N \neq 1$ be a maximal $A$-invariant normal subgroup of $G$. As $N$ is soluble by step 1 , we have that $G / N$ must be non-soluble. By Lemma 2.3 , we write $G / N=S_{1} \times \cdots \times S_{t}$, where $S_{i}$ are isomorphic non-abelian simple groups, and $\mathbf{C}_{G / N}(A) \cong \mathbf{C}_{S}(B)$, where $S=S_{1}$ and $B=\mathbf{N}_{A}(S)$. Firstly, we prove that the action of $B$ on $S$ is non-trivial. Suppose on the contrary that the action is trivial. Since $C /(C \cap N) \cong \mathbf{C}_{G / N}(A) \cong \mathbf{C}_{S}(B)=S$, by Lemma 2.6, it follows that every Sylow 2-subgroup $P$ of $S$ is cyclic or dihedral. As $S$ is nonabelian simple, the first case cannot happen. Then $P$ must be dihedral and, by Lemma 2.4(b), $S$ must be isomorphic to $\operatorname{PSL}(2, q)$, with $q>3$ odd, or $\mathrm{A}_{7}$. As a consequence, $G$ has a composition factor isomorphic to one of such groups. This contradiction proves that $B$ acts non-trivially on $S$. Thus we apply Lemma 3.1 and obtain that $S$ is simple of Lie type, $S=S\left(q^{t}\right)$, and is defined over the field with $q^{t}$ elements where $t=\left|B / \mathbf{C}_{B}(S)\right|$. Also, $\mathbf{C}_{S}(B)=S(q)$ has the same Lie type. By applying Lemma 2.1 , we have $\mathbf{C}_{G / N}(A) \cong C /(C \cap N) \cong \mathbf{C}_{S}(B)$, so we deduce that a Sylow 2-subgroup $T$ of $\mathbf{C}_{S}(B)$ is cyclic or dihedral. Suppose first that $T$ is cyclic. In this case $\mathbf{C}_{S}(B)$ cannot be non-abelian simple, and by Remark 2.7 , we get $\mathbf{C}_{S}(B) \cong \operatorname{PSL}(2,2)$ or $\operatorname{Sz}(2)$. Hence $S \cong \operatorname{PSL}\left(2,2^{t}\right)$ or $\mathrm{Sz}\left(2^{t}\right)$, contradicting the hypotheses. If $T$ is dihedral, then the minimality implies that $S$ is soluble. This contradiction completes the proof of this step.

Step 3. Final contradiction.

By Lemma 2.3, we can write $G=H_{1} \times \cdots \times H_{n}$, where the subgroups $H_{i}$ are isomorphic simple groups, and $C \cong \mathbf{C}_{H}(B)$, where $H=H_{1}$ and $B=\mathbf{N}_{A}(H)$. Also, $H$ must be non-abelian. The hypotheses imply that $\mathbf{C}_{H}(B)$ has a dihedral Sylow 2-subgroup. If $H<G$, the minimality implies that $H$ is soluble, a contradiction. For this reason, $G$ must be non-abelian simple. Then Lemma 3.1 shows that $G$ is of Lie type, say $G=G\left(q^{r}\right)$, defined over the finite field with $q^{r}$ elements, $q$ being a prime power and $r=\left|A / \mathbf{C}_{A}(G)\right|$. Furthermore, $C=G(q)$. Now, the Sylow 2-subgroups of $C$ are dihedral. Among the groups listed in Remark 2.7 none has dihedral Sylow 2-subgroups, except the group $\operatorname{PSL}(2,3)$ (whose Sylow 2 -subgroup is $C_{2} \times C_{2}$ ). This case gives $G \cong \operatorname{PSL}\left(2,3^{r}\right)$, which leads to a contradiction. Finally, if $C$ is non-abelian simple, then Lemma 2.4(b) yields $G \cong \operatorname{PSL}\left(2, q^{r}\right)$, with $q^{r}>3$ odd, or $G \cong \mathrm{A}_{7}$, which contradicts the hypotheses too.

\section{Acknowledgements}

The authors would like to thank the referee for helpful comments and suggestions. This work was done while the second author was visiting the University Jaume I of Castellón, Spain. He would like to express his deep gratitude for the 
warm hospitality that he received in the Department of Mathematics. The first author is partially supported by the Valencian Government, Proyecto PROMETEOII/2015/011 and also by Universitat Jaume I, grant P11B2015-77. The second author is supported by the NNSF of China (No. 11301218) and the Nature Science Fund of Shandong Province (No. ZR2014AM020).

\section{References}

[1] Beltrán A. Invariant Sylow subgroups and solvability of finite groups. Arch. Math., 2016, 102 (2): 101-106.

[2] Brauer R. and Suzuki M., On finite groups of even order whose 2-Sylow group is a quaternion group. Proc. Nat. Acad. Sci. U. S. A., 1959, 45: $1757-1759$.

[3] Conway J.H., Curtis R.T., Norton S.P., Parker R.A., Wilson R.A. Atlas of Finite Groups. Oxford Univ. Press, London, 1985.

[4] Gorenstein D. Finite Groups. Chelsea Pub. Co., New York, 1980.

[5] Gorenstein G. Walter J.H. On finite groups with dihedral Sylow 2subgroups. Illinois J. Math. 1962, 6: 553-593.

[6] Kurzweil H., Stellmacher B. The theory of finite groups. An introduction. Springer-Verlag, Berlin-Heidelberg-New York, 2004.

[7] Chen Z.M., Wang Y.M. Minimal non-solvable groups with an acting group (in Chinese). Chinese Sci. Bull., 1989, 34 (22): 1691-1693.

[8] Walter J.H. The Characterization of Finite Groups with Abelian Sylow 2Subgroup. Ann. Math. 1969, 89: 405-514. 